\section{RELIEVE}

ISSN: 1134-4032
e-Journal of Educational Research,

Assessment and Evaluation

\title{
Actitudes hacia la estadística en estudiantes de Ciencias de la Educación. Propiedades psicométicas de la versión española del Survey of Attitudes Toward Statistics (SATS-36)
}

\author{
Attitudes towards statistics of education Sciences students. Psychometric properties of the \\ Spanish version of the Survey of Attitudes Towards Statistics (SATS-36)
}

\section{Rodríguez-Santero, Javier \& Gil-Flores, Javier}

Universidad de Sevilla

\begin{abstract}
The study of attitudes towards statistics is a topic which has aroused the interest of researchers in different countries and at teaching levels. In this work we present an analysis of the psychometric properties, in terms of structural validity and internal consistency, of a Spanish version of the Survey of Attitudes Towards Statistics (SATS-36) administered to education students. The results obtained from the 409 participants indicate a lack of fit for the six-factor model proposed by the authors of the SATS, especially compared with the five-factor model with corrected mistakes that the authors of this work propose. These results coincide with what has been defended by other investigations as to the reduction of the number of attitudinal components measured with the SATS
\end{abstract}

Keywords: Psychometrics, factor analysis, student attitudes, statistics, university

\footnotetext{
Resumen

El estudio de las actitudes hacia la estadística es un tópico que ha suscitado el interés de investigadores en diferentes países y niveles de enseñanza. En el trabajo aquí presentado se expone un análisis de las propiedades psicométricas, en términos de validez estructural y consistencia interna, de la aplicación, en el contexto universitario nacional, del conocido Survey of Attitudes Toward Statistics (SATS), en su versión de 36 ítems. Los resultados obtenidos a partir de los 409 participantes, indican falta de ajuste para el modelo de seis factores propuestos por los autores del SATS, sobre todo, en comparación con el modelo de cinco factores con errores corregidos que proponen los autores de este trabajo. Estos resultados coinciden con lo defendido por otras investigaciones en cuanto a la reducción del número de componentes actitudinales medidos con el SATS.
} Reception Date 2018 June 27

Approval Date 2019 March 05

Publication Date: 2019 March 05

Palabras clave: Psicometría, análisis factorial, actitudes de los estudiantes, estadística, universidad

La estadística constituye una herramienta útil para desenvolverse en un mundo en el que los datos numéricos y su tratamiento están presentes en muy diversos ámbitos. Más allá de una alfabetización estadística necesaria en los ciudadanos, la competencia estadística 
resulta imprescindible en la formación de profesionales de diferentes campos. En la educación superior la estadística es una materia transversal, incluida en los planes de estudios diseñados para grados adscritos a diferentes áreas de enseñanza universitaria. Las técnicas estadísticas suelen abordarse en materias cursadas en los primeros años, y buena parte del alumnado ha de recurrir a ellas en el momento de realizar sus trabajos finales de grado, frecuentemente enfocados hacia estudios empíricos en los que se recogen datos de naturaleza cuantitativa que requieren ser analizados.

Las actitudes de los estudiantes hacia las materias objeto de estudio es un tópico que ha mantenido el interés de los investigadores de manera sostenida en el tiempo. La razón se encuentra en el hecho de que las actitudes constituyen un factor clave para el aprendizaje. Una actitud positiva predispone al esfuerzo, a la implicación en el trabajo y contribuye al éxito académico (Bourne, 2018). En cambio, las actitudes de signo negativo constituyen uno de los mayores obstáculos para el aprendizaje (Waters, Martelli, Zakrajsek \& Popovich, 1988) y pueden llevar al alumnado al desinterés, la inhibición y el bloqueo ante el estudio, alcanzándose peores resultados (Blanco, 2008) y condicionando incluso la elección de carrera en quienes se disponen a iniciar estudios universitarios en el ámbito de las Ciencias Sociales (Bourne \& Nesbit, 2018). En este sentido, Posiblemente sean las actitudes hacia las materias de contenido lógico-matemático o científico las que en mayor medida han centrado y continúan centrando la atención de los investigadores (Haladyna \& Shaughnessy, 1982; Ma \& Kishor, 1997; Osborne, Simons \& Collins, 2003). En particular, el estudio de las actitudes hacia la estadística entre el alumnado universitario constituye un tópico ampliamente abordado en la literatura científica, que no ha perdido vigencia tanto dentro como fuera del contexto español, como reflejan los trabajos recientes de Carillo, Galy, Guthrie y Vanhems (2016), Comas, Martins, Nascimento y Estrada (2017), Ruiz de Miguel
(2015), Sulieman (2015), Vilá y Rubio (2016) o Walker y Brakke (2017).

La enseñanza de los conceptos estadísticos plantea especiales problemas didácticos en ámbitos disciplinares donde el alumnado no posee una base matemática tan amplia como la que caracteriza a estudiantes de carreras científicas (Blanco, 2008). Muchos alumnos suelen llegar a las asignaturas de estadística con preconcepciones y actitudes negativas hacia la materia, asociadas habitualmente con niveles altos de ansiedad cuando se enfrentan a las clases, ejercicios o exámenes de estas asignaturas (Carmona, 2004). Ello supone un problema de cara a los procesos de enseñanza, dada la potencial relación entre aprendizaje y actitud hacia la materia de estudio (Estrada, 2002).

Onwuegbuzie y Wilson (2003) clasifica las razones o causas que dan lugar a actitudes negativas hacia la estadística en tres categorías: situacionales, disposicionales y ambientales. Entre las razones situacionales se encuentran el conocimiento estadístico previo. Esta experiencia parte del conocimiento adquirido a partir de la vida cotidiana fuera del aula, fundamentalmente a través de la prensa o en los medios de comunicación (Gal y Ginsburg, 1994). Otra causa situacional se concreta en las asignaturas de estadística cursadas, es decir las experiencias previas de aprendizaje en contexto escolar (Comas, Martins, Nascimento \& Estrada, 2017). Las causas disposicionales hacen referencia a las conductas y aptitudes del alumnado, con especial relevancia del autoconcepto en materias de contenido matemático. Por último, Onwuegbuzie y Wilson (2003) incluye causas ambientales relacionadas con el contexto cultural y las características sociodemográficas de los estudiantes.

El interés por las actitudes hacia la estadística radica en la predisposición negativa con que muchos estudiantes universitarios se enfrentan a esta materia, al no reconocer su valor y sentirse incómodos ante el desafío que supone su estudio. En determinadas titulaciones, mayoritariamente nutridas de alumnado que cursó Bachillerato 
de Humanidades y Ciencias Sociales, una buena parte de este alumnado suele encontrar dificultad para el aprendizaje de los conceptos estadísticos. Como afirman Peter, Smith, Middledorp, Karpin, Sin y Kilgore (2013), la estadística puede resultar abstracta, requiere razonamiento lógico, pensamiento crítico, habilidades para la interpretación y la extracción de conclusiones. Todo ello genera percepciones de dificultad que reducen las expectativas de éxito en el aprendizaje. Las dificultades percibidas por los estudiantes pueden ir acompañadas de desinterés, estrés, ansiedad $y$, en general, actitudes poco adecuadas para abordar el estudio de la estadística.

Las relaciones entre actitudes hacia la estadística y rendimiento han sido analizadas en numerosos trabajos científicos, mostrando en la mayor parte de los casos que una actitud positiva se asocia a un mayor rendimiento de los estudiantes. El meta-análisis llevado a cabo por Emmioglu y Capa-Aydin (2012) sobre estudios desarrollados entre 1998 y 2011 se circunscribió a trabajos que utilizaron el SATS como instrumento de medida de las actitudes del alumnado universitario. Hallaron una relación positiva significativa entre ambas variables, con tamaños del efecto globales que oscilaban entre 0,20 y 0,30 dependiendo de cuál sea el componente de las actitudes en que se centre la atención. Algunos trabajos han abordado el análisis del rendimiento académico en estadística construyendo modelos de ecuaciones estructurales en los que las actitudes hacia la estadística se incluyen junto a variables como ansiedad, rendimiento previo o habilidades matemáticas, entre otras (Chiesi \& Primi, 2010; Hood, Creed \& Neumann, 2012; Sesé, Jiménez, Montaño \& Palmer, 2015; Tempelaar, van der Loeff \& Gijselaers, 2007). En estos modelos, las actitudes del alumnado universitario han resultado ser la principal o una de las principales variables que predicen el rendimiento de los estudiantes universitarios.

Las dificultades encontradas por el alumnado en el estudio de la estadística, junto al valor de las actitudes de cara al aprendizaje de esta materia (Gal \& Ginsburg, 1994; Ramirez, Schau \& Emmioglu, 2008), justifican la necesidad de medir las actitudes. La información sobre actitudes hacia la estadística permitiría identificar alumnado que potencialmente está en riesgo de fracaso y, en consecuencia, desarrollar una intervención preventiva que le ayude a superar la asignatura. Entre los numerosos instrumentos diseñados para medir las actitudes hacia la estadística se encuentran el Statistics Attitudes Survey (Roberts \& Bilderback, 1980), la escala Attitudes Toward Statistics (Wise, 1985), la escala Students'Attitudes Toward Statistics (Sutarso, 1992), el Survey of Attitudes Toward Statistics (Schau, Stevens, Dauphinee, \& Del Vecchio, 1995; Schau, 2003) o la Students' Attitudes Toward Statistics and Technology Scale (Anastasiadou, 2011). El primer instrumento creado para el contexto español fue la Escala de Actitudes hacia la Estadística, de Auzmendi (1991), aunque una clara tendencia seguida por los investigadores en este campo ha sido la utilización de versiones en castellano de las escalas generadas en el ámbito anglosajón, como quedó reflejado en las revisiones realizadas por Carmona (2004) o Blanco (2008).

De todos los instrumentos mencionados, probablemente el más utilizado durante las dos últimas décadas es el Survey of Attitudes Toward Statistics (SATS), de Schau et al. (1995). En su primera versión consta de 28 ítems o enunciados ante los que se responde utilizando una escala tipo likert, con siete grados que van desde el completo desacuerdo al completo acuerdo. Su construcción se basa en un concepto multidimensional de las actitudes hacia la estadística, que incluye cuatro factores referidos a afecto (seis ítems referidos a sentimientos positivos o negativos hacia la estadística), competencia cognitiva (seis ítems sobre percepciones de los estudiantes sobre sus conocimientos $y$ habilidades para aprender estadística), valor (nueve ítems que aluden a percepciones sobre la utilidad, relevancia y valor de la estadística en la vida personal y profesional) y dificultad (siete ítems referidos a consideraciones sobre 
la dificultad que entraña la estadística como materia). Posteriormente, se diseñó una versión de 36 ítems (Schau, 2003) añadiendo los factores esfuerzo (percepción sobre el trabajo invertido en aprender estadística) e interés (nivel de interés del estudiante en la estadística), con cuatro ítems cada uno de ellos. Schau y Emmioğlu (2012) han informado sobre la fiabilidad de la escala, que se cifra en valores adecuados de $\alpha$ de Cronbach: afecto (.81), competencia cognitiva (.84), valor (.87), dificultad (.76), interés (.89) y esfuerzo (.81).

Los dos nuevos factores añadidos en la versión de 28 ítems se corresponden con nuevos componentes del modelo de valorexpectativa que Wigfield y Eccles (2000) proponen para explicar la motivación hacia el rendimiento, y que fue adaptado al dominio estadístico y usado como base en la construcción del SATS. Tanto para la versión de 28 ítems como para la de 36, sus autores ofrecen dos modalidades pretest y postest, aplicables respectivamente al inicio 0 al término de un curso sobre estadística. Para las dos modalidades, los ítems presentan un contenido similar y se diferencian fundamentalmente en el tiempo verbal empleado.

El SATS ha sido utilizado para medir las actitudes del alumnado hacia la estadística en múltiples contextos, incluyendo la enseñanza secundaria, diferentes titulaciones universitarias y diferentes países. Parte de los estudios desarrollados han confirmado la estructura propuesta por sus autores, tanto para el SATS-28 (Bechrakis, Gialamas, \& Barkatsas, 2011; Chiesi \& Primi, 2009; Dauphinee, Schau, \& Stevens, 1997; Hilton, Schau, \& Olsen, 2004) como para el SATS-36 (Carillo et al. 2016; Coetzee \& Merwe, 2010; Stanisavljevic, Trajkovic, Marinkovic, Bukumiric, Cirkovic, \& Millic, 2014; Tempelaar, Schim, \& Gijselaers, 2007). Sin embargo Nolan, Beran y Hecker (2012), al revisar las características psicométricas encontradas en diferentes aplicaciones de una quincena de instrumentos utilizados para la medición de actitudes hacia la estadística, concluyen que la estructura de ambas versiones del SATS resulta algo conflictiva, en parte debido a las moderadas o altas correlaciones entre las subescalas. Otro problema se encuentra en que los análisis confirmatorios realizados en los trabajos que respaldan empíricamente la estructura del SATS han recurrido al agrupamiento de ítems (parcelling) dentro de cada factor, siguiendo el procedimiento empleado por Schau et al. (1995). Utilizando análisis factorial exploratorio y considerando los ítems individuales, Cashin y Elmore (2005) encontraron dos dimensiones en el SATS-28, al agruparse en una sola dimensión los factores afecto, competencia cognitiva y dificultad. Con la misma metodología, para una versión castellana de este instrumento, Estrada (2007) obtuvo una solución de cinco factores, en la que se constata una agrupación clara para el factor valor, mientras que los ítems relativos a afecto, competencia cognitiva y dificultad aparecen mezclados en los cuatro factores restantes.

En lo que respecta al SATS-36, las discrepancias que diferentes autores han encontrado con la estructura factorial propuesta son notorias, particularmente al utilizar ítems individuales en lugar de agrupados $\mathrm{o}$ al desarrollar adaptaciones a otros contextos. VanHoof, Kuppens, Castro, Verschaffel y Onghena (2011) aplicaron este instrumento a universitarios belgas, encontrando que la estructura de seis factores podría quedar reducida a cuatro, tras eliminar algunos ítems que mostraban un mal funcionamiento y combinar de nuevo en un solo factor las subescalas afecto, competencia cognitiva y dificultad. En cambio Khavenson, Orel y Tryakshina (2012), mediante análisis factorial exploratorio, hallaron siete factores al aplicar una adaptación rusa del SATS-36 dirigida a estudiantes universitarios, con una definición de factores y distribución de ítems que presentaba diferencias respecto a la estructura original. En su propuesta final de una versión rusa del instrumento, excluyeron el séptimo factor debido a que solo incluía dos ítems y registraba un bajo índice de fiabilidad medida por a de Cronbach. Tampoco ha sido confirmada la estructura del 
SATS-36 al aplicarlo a una población de estudiantes universitarios paquistaníes (Ashrafi \& Tariq, 2015). Recientemente, Hommik y Luik (2017) han adaptado el instrumento para su utilización con estudiantes estonios de educación secundaria; la falta de ajuste en el modelo original de seis factores ha llevado a estos autores a eliminar nueve ítems que presentaron un mal funcionamiento y plantear una estructura de cuatro factores que ha resultado ser más adecuada en términos de fiabilidad y validez.

Aunque el SATS-36 ha sido aplicado a muestras de estudiantes de distintas áreas de enseñanza universitaria en muy diversos países y se han analizado sus características técnicas, no hemos encontrado en la literatura ningún estudio de validación para ser utilizado con alumnado español. El objetivo del presente trabajo es comprobar las propiedades psicométricas, en términos de validez estructural y consistencia interna, de la versión española del SATS-36, cuando es aplicado en Ciencias de la Educación. Para este alumnado, la estadística suele ser una materia académica que entraña dificultad. Medir las actitudes hacia la estadística en este colectivo es interesante de cara a plantear la docencia y el aprendizaje de los contenidos estadísticos. En consecuencia, con este trabajo se pretende proponer un instrumento validado para la medición de las actitudes hacia la estadística en el contexto universitario español, particularmente en el ámbito de las Ciencias de Educación.

\section{Método}

\section{Participantes}

Han participado en el estudio un total de 409 estudiantes (55 hombres y 354 mujeres) de la Universidad de Sevilla, pertenecientes a tres cohortes de alumnado que cursaron una asignatura introductoria a la estadística, enmarcada en el plan de estudios del Grado en Pedagogía. Asumiendo que no hay razones para sospechar que las actitudes hacia la estadística del alumnado difieran significativamente en función de la universidad en la que cursan sus estudios de Ciencias de la Educación, la muestra participante se ha tomado de la Universidad de Sevilla, por razones de accesibilidad. Las edades de los participantes se encuentran comprendidas entre los 19 y los 54 años, si bien el 72,9\% no cuenta con más de 23 años. $\mathrm{Su}$ experiencia previa en estadística es variada, teniendo en cuenta las modalidades de Bachillerato cursadas previamente: el 2,4\% ha cursado Bachillerato de Artes, el 68,5\% de Humanidades y Ciencias Sociales, el 3,2\% de Tecnología, el $19,6 \%$ de Ciencias de la Naturaleza y la Salud y el 6,4\% no ha cursado Bachillerato, por haber accedido a la universidad a través de otras vías como la Formación Profesional o las pruebas para mayores de 25 años. Dada la diversidad de trayectorias académicas previas, el número de materias de contenido matemático o estadístico cursadas con anterioridad varía entre 0 y 5 , si bien el $57,8 \%$ de los participantes ha cursado como máximo una materia y el 91,6\% no cursó más de dos.

\section{Instrumento}

La versión española del SATS-36 se construyó partiendo de la ya utilizada para el SATS-28 en el trabajo de Estrada (2007). Los ocho ítems añadidos fueron traducidos por los autores, sometiéndolos posteriormente a la revisión de un traductor profesional. Una primera redacción de los 36 ítems fue sometida a la consideración de varios estudiantes, con el fin de identificar posibles ambigüedades o formulaciones difíciles de comprender. Esto llevó a introducir ajustes en la versión final del instrumento (véase https://goo.gl/3oVUpN).

El SATS-36 fue administrado a los participantes al iniciarse la asignatura, que es cursada en el segundo año de los estudios correspondientes al Grado en Pedagogía y se ubica temporalmente en el segundo cuatrimestre del año académico. De este modo, la recogida de datos tuvo lugar en el mes de febrero de 2016 (152 estudiantes), 2017 (166 estudiantes) y $2018 \quad$ (91 estudiantes). La escala fue respondida en la primera sesión de clase, antes de presentar la asignatura y dar a conocer el programa docente propuesto para la misma. De este 
modo, las actitudes del alumnado no se vieron influenciadas por la experiencia adquirida durante el proceso de aprendizaje de la asignatura.

\section{Análisis de datos}

Las respuestas obtenidas para cada ítem del SATS-36 expresan el grado de acuerdo con su enunciado, con valores de 1 a 7 . De cara al análisis, las respuestas para los 19 ítems con enunciados negativos fueron codificadas de manera inversa, de tal forma que al máximo acuerdo con el ítem se asignó el valor 1 en lugar de 7. De ese modo, las actitudes positivas hacia la estadística se corresponden con las puntuaciones altas en los ítems.

El análisis factorial confirmatorio (en adelante AFC), practicado sobre la estructura de seis factores defendida por los autores del instrumento, arrojó niveles bajos en cuanto a la bondad de ajuste del modelo, razón por la que los autores del presente trabajo decidieron plantear un nuevo modelo. Para ello, en primera instancia se efectuó un análisis factorial exploratorio por componentes principales y rotación varimax. Teniendo en cuenta que las variables presentan en general comunalidades altas, el método de componentes principales permite obtener estimadores insesgados. Para comprobar la estructura factorial resultante del AFE, se ha realizado un AFC, empleando el método de máxima verosimilitud. Finalmente, hemos recurrido a los valores del $\alpha$ de Cronbach y $\Omega$ de McDonald para estimar la fiabilidad de cada una de las dimensiones consideradas.
Para todos los análisis se ha utilizado el software SPSS y AMOS en su versión 24, salvo en el caso del coeficiente $\Omega$ de McDonald, calculado a partir de Excel.

\section{Resultados}

\section{Análisis exploratorio}

Para asegurar la viabilidad del análisis factorial se ha valorado la matriz de correlaciones entre ítems. La prueba de esfericidad de Bartlett $\left(\chi^{2}=5008,800\right.$; gl = 435; $\mathrm{p}<.000$ ) permite afirmar que dicha matriz no es una matriz identidad, al tiempo que la medida de adecuación de muestreo de KaiserMeyer-Olkin $(\mathrm{KMO}=.875)$ alcanza un valor elevado. Ambos resultados indican que es posible la extracción de factores a partir de la matriz de correlaciones observadas.

Siguiendo el criterio habitual de retener factores cuyo autovalor es al menos la unidad, el AFE permitió fijar una estructura de cinco factores (Affect-competence, Value, Difficulty, Interest, Effort). Fueron eliminados previamente seis ítems $(3,6,8,10$, 11 y 31) que o bien no presentaban un nivel adecuado de saturación $(r>.40)$ en ninguno de los factores, o saturaban con nivel alto en más de uno de ellos. Esta estructura es capaz de explicar el 56.12\% de la varianza total. De eliminar solo algunos de los seis ítems mencionados, el porcentaje de varianza explicado sería inferior. En la Tabla 1 se recoge la distribución de ítems por cada uno de los cinco factores identificados. 
Tabla 1 - Matriz de componentes rotados para los ítems de la escala

Componente Interest $\begin{gathered}\text { Affect- } \\ \text { competence }\end{gathered}$ Value Effort Difficulty

20. Estoy interesado/a en usar la estadística

19. Disfrutaré de las clases de estadística

29. Estoy interesado/a en aprender estadística

\begin{tabular}{|c|c|c|c|c|}
\hline ,789 & 164 & 318 & 132 & ,027 \\
\hline ,696 & 273 & ,043 & ,061 & 068, \\
\hline ,666 & 136 & 186 & 286 &,- 075 \\
\hline ,644 & 182 & ,144 & ,272 &,- 201 \\
\hline ,636 & ,095 & 220 & ,032 &,- 040 \\
\hline ,558 & ,003 & 182 &,- 125 & 102 \\
\hline ,028 & ,769 & 109 & ,029 &,- 056 \\
\hline ,023 & ,668 & 118 &,- 069 &,- 011 \\
\hline ,041 & ,647 & 255 &,- 052 & ,038 \\
\hline 253, & ,631 & ,116 &,- 219 & 259, \\
\hline 253, & ,608 & ,053 & ,012 & 420 \\
\hline 108 & ,606 & ,013 & ,139 & 293, \\
\hline 267 & ,564 & ,204 &,- 062 & 259, \\
\hline 310 & ,541 & ,048 & 151 &,- 100 \\
\hline 136 & 122 & ,737 & ,001 &,- 022 \\
\hline 131 & ,038 & ,717 & ,062 &,- 080 \\
\hline 136 & ,111 & ,685 & ,010 & 196, \\
\hline 349, & 162 & ,639 & ,092 & 182 \\
\hline ,018 & ,248 & ,596 & 112 &,- 078 \\
\hline ,375 & ,086 &, 590 &,- 054 & ,131 \\
\hline ,364 & 132 & ,480 & 191 &,- 176 \\
\hline 106 &,- 017 & ,058 & ,827 & 019, \\
\hline 151 &,- 032 &,- 018 & ,721 &,- 061 \\
\hline -,007 & ,004 &,- 001 & ,671 &,- 109 \\
\hline ,068 & ,003 & ,207 & ,643 &,- 100 \\
\hline ,030 &,- 038 &,- 097 &,- 043 & ,728 \\
\hline,- 044 & ,080 & ,065 &,- 285 & 614 \\
\hline,- 123 & ,077 & ,077 &,- 024 & ,551 \\
\hline,- 069 & ,202 & ,102 & ,047 & ,511 \\
\hline 291 & ,059 &,- 110 &,- 121 & ,468 \\
\hline
\end{tabular}

23. Estoy interesado/a en entender la información estadística 


\section{Análisis confirmatorio}

Con el fin de valorar comparativamente los resultados obtenidos al considerar cinco factores, se propone un primer modelo que coincide con la estructura de seis factores defendida por los autores del SATS-36. En el segundo modelo se incluyen los cinco componentes, considerando que cada ítem carga únicamente sobre una variable latente, que los factores covarían y que los términos de error no están correlacionados. En un tercer modelo se incluyen, junto a los cinco factores comunes, las correlaciones entre errores (EC), aunque solo para las cinco parejas de ítems que presentaban los índices de modificación más altos (4-5, 9-33, 19-20, 19-23 y 23-29).

La Figura 1 muestra gráficamente la estructura del modelo final (cinco factores $\mathrm{EC)}$.

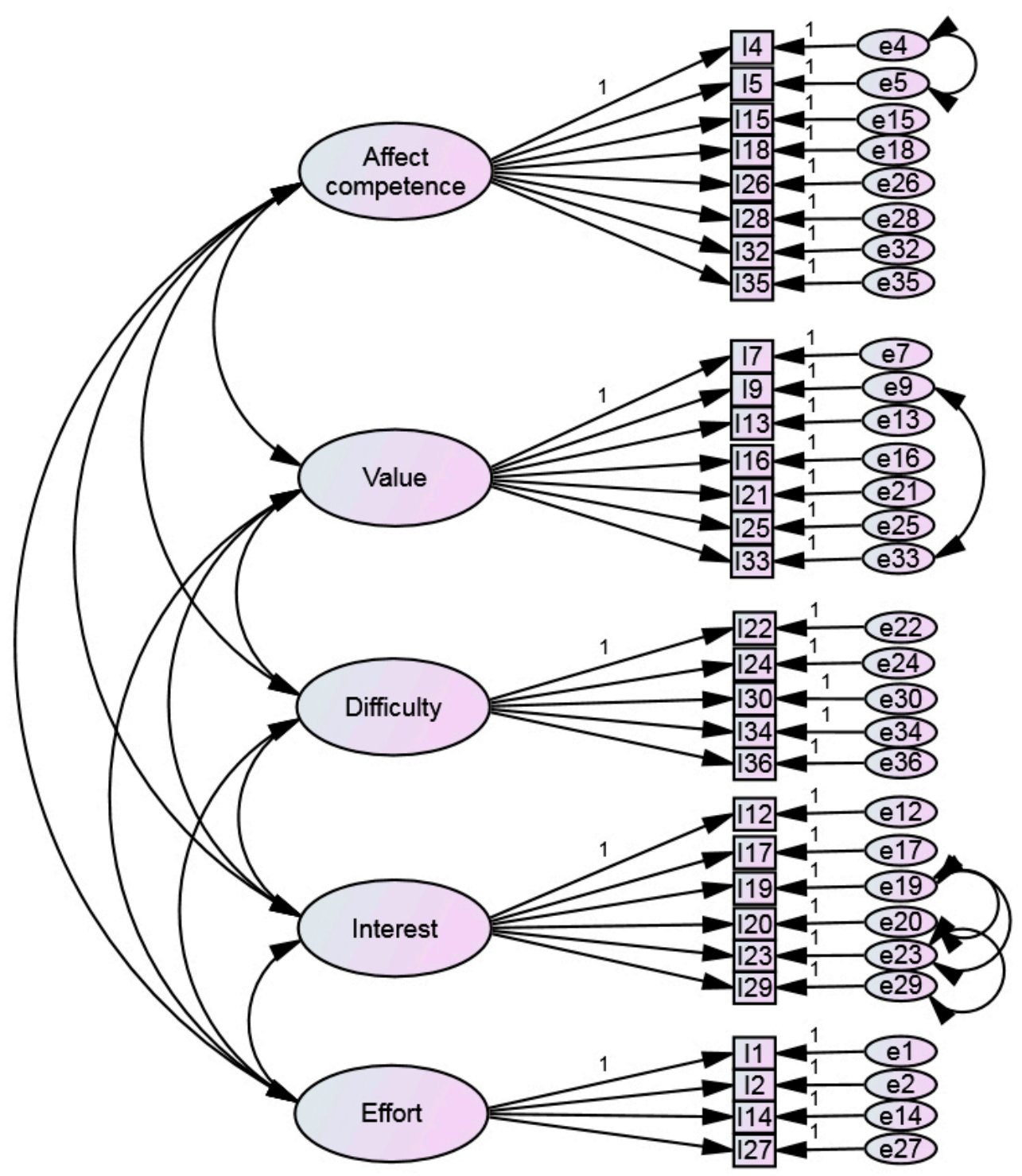

Figura 1. Modelo factorial para el SATS.

Atendiendo a los índices de modificación, se valoró la significación de los parámetros correspondientes a covarianzas entre factores únicos. Para valorar la bondad de ajuste de cada uno de los tres modelos, se ha considerado, tomando como referencia a
Boomsma (2000), Hu y Bentler (1999), Marsh, Hau, y Wen, (2004) y Ntoumanis, (2001), la razón entre $\chi^{2}$ y los grados de libertad, el índice de ajuste comparativo (CFI), el índice de bondad de ajuste (GFI), el índice de error de cuadrático medio (RMR) y 
el error cuadrático medio de aproximación (RMSEA). Estos son considerados aceptables cuando $\chi 2 /$ d.f. es inferior a 5 , los índices CFI y GFI son superiores a .85 (lo ideal es por encima de .90) y los índices de error (RMR y RMSEA) son inferiores a .07, aunque lo ideal sería por debajo de .05 .

Los resultados mostrados en la Tabla 2 indican falta de ajuste para el modelo de seis factores propuesto por los autores del SATS36. Basándonos en los criterios mencionados anteriormente, la bondad de ajuste es sensiblemente mejor para el modelo final de cinco factores con EC. En este modelo, la razón entre $\chi^{2}$ y los grados de libertad se sitúa en 2.19, valor que no supera el límite de 3 y supone, por tanto, un buen ajuste entre el modelo propuesto y los datos observados. En cambio, este índice superaba el umbral de 3 en el caso del modelo original. En cuanto al índice de ajuste comparativo (CFI) y el índice de bondad de ajuste (GFI), en el modelo final se obtienen las mejores aproximaciones a la cota de .900 , valor a partir del cual puede considerarse un óptimo nivel de ajuste. El valor de RMR, aunque no se mantiene por debajo de .050, presenta un mejor comportamiento en el modelo de cinco factores con EC que en los otros dos modelos. Además, en el modelo final el error cuadrático medio de aproximación (RMSEA) resulta muy adecuado, pues ronda el valor .050 .

Tabla 2 - Índices de bondad de ajuste para los modelos factoriales

\begin{tabular}{lccccc}
\hline Modelo & $\chi^{2} /$ d.f. & CFI & GFI & RMR & RMSEA (IC 90\%) \\
\hline Modelo original & 3.02 & .767 & .770 & .142 & $.070(.067-.074)$ \\
Cinco factores & 2.42 & .854 & .860 & .117 & $.059(.054-.064)$ \\
Cinco factores con EC & 2.19 & .878 & .873 & .115 & $.055(.049-.059)$ \\
\hline
\end{tabular}

Nota: CFI = Comparative Fit Index; GFI = Goodness of Fit Index; RMR = Root Mean square Residual; RMSEA = Root Mean Square Error of Approximation

Se han estudiado también los coeficientes de correlación estandarizados entre factores y entre variables y factores, con objeto de confirmar, por un lado, la validez de constructo del instrumento $\mathrm{y}$, por otro, la validez discriminante del mismo, prestando especial atención, en este último caso, a si la correlación entre las variables latentes, atenuadas por el error de medición (+/- 2 veces el error de medición), era inferior a la unidad. La mayor parte de las cargas factoriales de cada variable alcanzaron valores elevados $(\mathrm{R}>$.6). Todas ellas resultaron estadísticamente significativas con $\mathrm{p}<.01$. La carga más baja corresponde al ítem 22 (La estadística es una materia que la mayoría de las personas aprende rápidamente), con un valor .406 en la subescala Difficulty. La más alta es .865, que se registra para el ítem 20 (Estoy interesado/a en usar la estadística) en la subescala Interest. Los resultados obtenidos apoyan, por tanto, la estructura de cinco factores propuesta para el instrumento.

La inexistencia de correlaciones entre factores próximas a la unidad (ver Tabla 3), nos hace descartar que dos factores representen en realidad una misma dimensión. Ello avala la validez discriminante del modelo, que presentaría dimensiones suficientemente diferenciadas. 
Tabla 3 - Matriz de correlaciones entre factores y entre ítems y factores

\begin{tabular}{|c|c|c|c|c|c|}
\hline & Interest & Difficulty & Value & Effort & $\begin{array}{c}\text { Affect- } \\
\text { competence }\end{array}$ \\
\hline Interest & 1.000 & & & & \\
\hline Difficulty & .019 & 1.000 & & & \\
\hline Value & .663 & .060 & 1.000 & & \\
\hline Effort & .325 & -.371 & .262 & 1.000 & \\
\hline Affect_competence & .440 & .561 & .457 & -.011 & 1.000 \\
\hline $\mathrm{I} 19-\mathrm{9}$ & .640 & .012 & .424 & .208 & .282 \\
\hline I7 & .321 & .029 & .484 & .127 & .221 \\
\hline I33 & .490 & .044 & .739 & .194 & .337 \\
\hline $\mathrm{I} 25$ & .413 & .037 & .623 & .163 & .284 \\
\hline I13 & .428 & .039 & .645 & .169 & .294 \\
\hline I9 & .404 & .036 & .609 & .160 & .278 \\
\hline I17 & .416 & .008 & .276 & .135 & .183 \\
\hline I12 & .608 & .012 & .404 & .197 & .268 \\
\hline I35 & .331 & .422 & .343 & -.008 & .752 \\
\hline $\mathrm{I} 32$ & .205 & .261 & .213 & -.005 & .466 \\
\hline $\mathrm{I} 28$ & .322 & .410 & .334 & -.008 & .731 \\
\hline I26 & .296 & .377 & .307 & -.007 & .672 \\
\hline $\mathrm{I} 27$ & .181 & -.206 & .146 & .557 & -.006 \\
\hline I14 & .197 & -.225 & .159 & .608 & -.006 \\
\hline I2 & .263 & -.301 & .213 & .811 & -.009 \\
\hline I1 & .233 & -.266 & .188 & .717 & -.008 \\
\hline I29 & .629 & .012 & .417 & .204 & .277 \\
\hline $\mathrm{I} 23$ & .641 & .012 & .425 & .208 & .282 \\
\hline $\mathrm{I} 20$ & .865 & .017 & .574 & .281 & .381 \\
\hline I36 & .008 & .427 & .026 & -.158 & .240 \\
\hline I34 & .010 & .499 & .030 & -.185 & .280 \\
\hline I30 & .009 & .453 & .027 & -.168 & .254 \\
\hline I24 & .012 & .621 & .037 & -.230 & .348 \\
\hline I22 & .008 & .406 & .024 & -.150 & .228 \\
\hline I21 & .387 & .035 & .584 & .153 & .267 \\
\hline I16 & .397 & .036 & .598 & .157 & .273 \\
\hline I18 & .318 & .405 & .330 & -.008 & .723 \\
\hline I15 & .256 & .326 & .266 & -.006 & .582 \\
\hline I5 & .242 & .309 & .251 & -.006 & .550 \\
\hline I4 & .207 & .264 & .215 & -.005 & .470 \\
\hline
\end{tabular}

\section{Fiabilidad}

La fiabilidad del instrumento (ver Tabla 4), estimada a partir de $\alpha$ de Cronbach, resulta buena $(\alpha>.80)$ para las dimensiones "Affectcompetence" y "Value" y aceptable $(\alpha>.70)$ para "Difficulty", "Interest" y Effort, de acuerdo con los criterios propuestos por George y Mallery (2003). En cuanto al coeficiente $\Omega$ de McDonald, los valores obtenidos podrían considerarse más que aceptables. Alcanzan o superan el .80, con la única excepción de la dimensión "Difficulty”, donde se sitúa en .73.
Tabla 4 - Cálculo de fiabilidad en cada factor

\begin{tabular}{lcc}
\hline Subescalas & $\begin{array}{c}\alpha \text { de } \\
\text { Cronbach }\end{array}$ & $\begin{array}{c}\Omega \text { de } \\
\text { McDonald }\end{array}$ \\
\hline Affect-competence & .81 & .83 \\
Value & .86 & .88 \\
Difficulty & .72 & .73 \\
Interest & .79 & .84 \\
Effort & .76 & .80 \\
\hline
\end{tabular}




\section{Discusión y conclusiones}

El objetivo de nuestro estudio ha sido valorar las propiedades psicométricas el SATS-36 al ser aplicado a una muestra de estudiantes españoles de Ciencias de la Educación, con la finalidad última de proponer una versión adaptada a este colectivo. Al analizar el instrumento, hemos considerado cada uno de los ítems, sin recurrir a la técnica de agrupamiento (item parcelling) propuesta por Schau et al. (1995) para el análisis de la estructura del SATS-28 y posteriormente utilizada en trabajos que han confirmado una estructura subyacente de 6 factores para el SATS-36 (Carillo et al., 2016; Coetzee \& Merwe, 2010; Tempelaar et al., 2007). El item parcelling consiste en formar dentro de cada dimensión grupos de 3 ó 4 ítems, considerando en los análisis factoriales exploratorios o confirmatorios la suma o las puntuaciones medias en estos grupos en lugar de las puntuaciones individuales. El objetivo es reducir problemas de normalidad en ciertos ítems y mejorar la fiabilidad. Sin embargo, este procedimiento ha suscitado controversia (Bandalos \& Finney, 2001), particularmente porque se asume que los ítems agrupados en parcelas miden la misma dimensión, lo cual no puede asumirse sin una verificación previa. Al optar por la utilización de ítems individuales y no parcelados, en el presente trabajo obtenemos resultados que se apartan en cierta medida de la estructura original del instrumento. Sin embargo, el análisis de los ítems individuales permite acceder a la verdadera estructura del conjunto de ítems que componen el SATS-36, evitando los sesgos derivados del agrupamiento de ítems (VanHoof et al., 2011).

Tras los análisis realizados, hemos suprimido ítems que mostraron un funcionamiento inadecuado, resultando un modelo de 30 ítems agrupados en 5 factores que suponen la integración de las subescalas afecto y competencia cognitiva en un solo factor. Este modelo resulta preferible para la versión española, dado que presenta medidas de bondad de ajuste más adecuadas que las obtenidas al considerar la estructura original de 36 ítems en 6 factores. Una conclusión de este trabajo es, por tanto, que con un modelo más parsimonioso de 5 factores se describen adecuadamente las interrelaciones observadas entre los ítems del instrumento. Los ítems incluidos en cuatro de las dimensiones del modelo -valor, dificultad, interés y esfuerzoson prácticamente coincidentes con los propuestos por Schau (2003) para el SATS36. La única salvedad es la ausencia de los ítems suprimidos y la presencia en la dimensión interés de los ítems 17 y 19, procedentes respectivamente de las dimensiones valor y afecto en la estructura original. La principal modificación se concreta en el agrupamiento de los factores afecto y competencia cognitiva en una sola dimensión, en la que quedan incluidos 8 ítems, de los cuales la mitad proceden de la dimensión afecto y la otra mitad de la dimensión competencia cognitiva.

Este resultado se alinea con la reducción del número de componentes actitudinales medidos con el SATS, ya observada en trabajos anteriores que consideraron ítems individuales y no parcelados. Un resultado reiterado ha sido el agrupamiento de las subescalas afecto, competencia cognitiva y dificultad, al que se llegó en los trabajos de VanHoof et al. (2011), con una adaptación del SATS-36, y de Cashin y Elmore (2005), trabajando con el SATS-28. Detrás de este agrupamiento se encuentra la existencia de correlaciones entre las referidas subescalas, ya sugerida por Nolan et al., (2012) para explicar las discrepancias respecto a la estructura original del instrumento. De hecho, la correlación entre los factores afecto y competencia cognitiva ha llegado a sobrepasar el valor 0.90 en diferentes estudios que analizaron la estructura del SATS-28 (Dauphinee et al., 1997; Hilton et al., 2004; Schau et al., 1995) y el valor 0.80 en otros focalizados en el SATS-36 (Tempelaar et al., 2007; VanHoof et al., 2011). En la versión española aplicada en nuestro estudio, la reducción de subescalas se ha concretado en el agrupamiento de los factores afecto y competencia cognitiva, permaneciendo dificultad como un componente diferenciado 
de las actitudes hacia la estadística. Las correlaciones entre estos 5 factores alcanzan valores situados por debajo de 0.67 .

En lo que respecta a la fiabilidad medida por $\alpha$ de Cronbach y $\Omega$ de McDonald, los 5 factores encontrados para la adaptación española del SATS-36 presentan valores comprendidos entre .72 y .88. Estos índices reflejan en general un buen nivel de consistencia interna para las subescalas, teniendo en cuenta que el número de ítems es relativamente bajo en algunas de ellas. En esta misma línea, autores como Tempelaar et al. (2007) o Carillo et al. (2016) obtuvieron valores de fiabilidad comprendidos entre $.76 \mathrm{y}$ .83 en el modelo original de 6 factores, con la excepción de la dimensión dificultad, a la que correspondieron valores $\alpha=.68$ y $\alpha=.59$ respectivamente en ambos trabajos.

De acuerdo con las propiedades psicométricas de la adaptación española del SATS-36, podemos afirmar que el instrumento propuesto es adecuado para la medición de las actitudes hacia la estadística en el alumnado universitario español que cursa Ciencias de la Educación. Disponer de un instrumento para medir las actitudes hacia la estadística tiene un indudable interés desde el punto de vista de la enseñanza y aprendizaje de esta materia. Teniendo en cuenta la relación entre las actitudes y los logros en términos de aprendizaje, el diagnóstico previo de las actitudes del alumnado que se dispone a iniciar un curso de estadística permitiría orientar el proceso de enseñanza y detectar estudiantes en los que es mayor el riesgo de fracaso o abandono. En este sentido, el carácter multidimensional del constructo actitudes proporciona una información diferenciada en la que basar la intervención del profesorado.

La situación observada en relación a cada una de las dimensiones medidas en el constructo actitudes, permitirá poner en práctica un enfoque pedagógico adaptado a los estudiantes (Carillo et al., 2016). Así, por ejemplo, si los déficits actitudinales se concretan en sentimientos negativos (dimensión afecto-competencia), será apropiado potenciar un enfoque lúdico capaz de hacer atractiva la materia. Si los estudiantes no aprecian la utilidad de la estadística (dimensión valor), habría que insistir en la importancia de este instrumento en el ámbito laboral, recurriendo por ejemplo al testimonio de profesionales, o en la vida cotidiana, mostrando por ejemplo estadísticas sobre temas de actualidad aparecidas en los medios de comunicación. Ante percepciones de que la estadística es compleja y requiere el uso de complicadas fórmulas (dimensión dificultad), la enseñanza habría de graduar la presentación de los contenidos comenzando en niveles fácilmente accesibles para el alumnado, y priorizar el uso de herramientas informáticas que liberen de la aplicación manual de cálculos basados en fórmulas.

La disponibilidad de un instrumento validado para el diagnóstico de las actitudes hacia la estadística en estudiantes universitarios representa además una contribución para la docencia y para el desarrollo de la investigación sobre este campo en el contexto español. El SATS-36 podrá incorporarse al repertorio de instrumentos disponibles en español (Blanco, 2008; Carmona, 2004), facilitando el trabajo de los investigadores interesados por temas tales como, por ejemplo, la incidencia de los diferentes métodos de enseñanza de la estadística sobre las actitudes, o la relación entre actitudes y rendimiento académico. Además, el profesorado contará con una herramienta más para identificar actitudes negativas en su alumnado y poner en marcha intervenciones dirigidas a mejorarlas $\mathrm{y}$ contribuir de ese modo al aprendizaje.

No obstante, nuestro estudio presenta limitaciones que deben ser tenidas en cuenta. La muestra utilizada, a pesar de tener un tamaño elevado, es homogénea y se circunscribe a un contexto académico concreto, pues se trata de alumnado que cursa estudios de Pedagogía. En consecuencia, las conclusiones alcanzadas tendrían que ser consideradas con cautela a la hora de su generalización. Por ese motivo, parece recomendable la realización de nuevos 
estudios con muestras de alumnado de otras titulaciones, que permitan comprobar la invarianza de la estructura aquí propuesta para los componentes de las actitudes hacia la estadística medidas con el SATS. Por otra parte, la estructura hallada para la versión pretest del SATS podría de ser objeto de confirmación en el caso de la versión pos-test utilizada para medir las actitudes del alumnado tras haber seguido un curso de estadística. Por último, la supresión de ítems que han mostrado un funcionamiento inadecuado, reduciendo la escala a 30 elementos, merecería un análisis más detallado. En este sentido, podría revisarse la redacción de algunos de estos ítems en la versión original, recurriendo a la valoración del contenido de los mismos por parte de los estudiantes, con el fin de identificar posibles ambigüedades o dificultades de interpretación que expliquen el problema. Esto permitiría analizar las propiedades psicométricas de una versión revisada del SATS-36, aplicándolo con carácter previo o posterior a cursar la asignatura de contenido estadístico.

\section{Referencias}

Anastasiadou, S. D. (2011). Reliability and validity testing of a new scale for measuring attitudes toward learning statistics with technology. Acta Didactica Napocensia, 4(1), 1-10.

Ashrafi, G. M., \& Tariq, M. I. (2015). Investigating discrepancies in reliability coefficient of survey of students ' attitudes towards statistics (SATS 36) for a Pakistani sample. International Interdisciplinary Journal of Education, 4(7), 201-225. doi: https://doi.org/10.12816/0025212

Auzmendi, E. (1991). Factors related to attitudes toward statistics: A study with a Spanish sample. Paper presented at the annual meeting of the American Educational Research Association, Chicago.

Bandalos, D. L., \& Finney, S. J. (2001). Item parceling issues in structural equation modeling. In G. A. Marcoulides and Schumacker, R. E. (Eds.), New Developments and Techniques in Structural Equation
Modeling (chap. 10, 269-296), Hillsdale, N.J.: Lawrence Erlbaum Associates.

Bechrakis, T., Gialamas, V., \& Barkatsas, A. N. (2011). Survey of Attitudes Toward Statistics (SATS): An investigation of its construct validity and its factor structure invariance by gender. International Journal of Theoretical Educational Practice, 1(1), 1-15.

Blanco, A. (2008). Una revisión crítica de la investigación sobre las actitudes de los estudiantes universitarios hacia la estadística. Revista Complutense de Educación, 19(2), 311-330.

Bollen, K. A. (1989). Structural equations with latent variables. New York: Wiley. doi: https://doi.org/10.1002/9781118619179

Boomsma, A. (2000). Reporting Analyses of Covariance Structures. Structural Equation Modeling: A Multidisciplinary Journal, 7(3), 461-483. doi: https://doi.org/10.1207/S15328007SEM0703_6

Bourne, V. J. (2018). Exploring Statistics Anxiety: Contrasting Mathematical, Academic Performance and Trait Psychological Predictors. Psychology Teaching Review, 24(1), 35-43.

Carillo, K., Galy, N., Guthrie,C., \& Vanhems, A. (2016). «J'aime pas les stats!» - Mesure et analyse de l'attitude à l'égard du cours de statistique dans une école de management. Statistique et enseignement, 7(1), 3-31.

Carmona, J. (2004). Una revisión de las evidencias de fiabilidad y validez de los cuestionarios de actitudes y ansiedad hacia la estadística. Statistics Education Research Journal, 3(1), 5-28.

Carmona, J., Martínez R.J. \& Sánchez, M. (2005). Mathematical background and attitudes toward statistics in a sample of Spanish college students. Psychological Reports, 97(1), 53-62. doi: https://doi.org/10.2466/pr0.97.1.53-62

Cashin, S. E., \& Elmore, P. B. (2005). The Survey of Attitudes Toward Statistics Scale: a construct validity study. Educational and Psychological Measurement, 65(3), 509-524. doi: https://doi.org/10.1177/0013164404272488 
Chiesi, F., \& Primi, C. (2009). Assessing statistics attitudes among college students: Psychometric properties of the Italian version of the Survey of Attitudes toward Statistics (SATS). Learning and Individual Differences, 19(2), 309-313. doi: https://doi.org/10.1016/j.lindif.2008.10.008

Chiesi, F., \& Primi, C. (2010). Cognitive and non-cognitive factors related to students statistics achievement. Statistics Education Research Journal, 9(1), 6-26. Retrieved from http://www.stat.auckland.ac.nz/ iase/serj/SER J9\%281\%29_Chiesi_Primi.pdf

Coetzee, S., \& Merwe, P. V. D. (2010). Industrial psychology students' attitudes towards statistics. SA Journal of Industrial Psychology, 36, 1-8. doi: https://doi.org/10.4102/sajip.v36i1.843

Cohen, J. (1988). Statistical power analysis for the behavioral sciences (2nd ed.). Hillsdale, N.J.: Lawrence Erlbaum.

Comas, C., Martins, J., Nascimento, M.M. y Estrada, A. (2017). Estudio de las actitudes hacia la estadística en estudiantes de Psicología. Bolema: Boletim de Educação Matemática, 31(57), 479-496. doi: https://doi.org/10.1590/1980-4415v31n57a23

Dauphinee, T. L., Schau, C., \& Stevens, J. J. (1997). Survey of Attitudes Toward Statistics: Factor structure and factorial invariance for women and men. Structural Equation Modeling, 4(2), 129-141. doi: https://doi.org/10.1080/10705519709540066

Estrada, A. (2002). Análisis de las actitudes y conocimientos estadísticos elementales en la formación del profesorado. Tesis doctoral. Universidad Autónoma de Barcelona

Estrada, A. (2007). Actitudes hacia la estadística: un estudio con profesores de Educación Primaria en formación y en ejercicio. En M. Camacho, P. Flores y P. Bolea (Eds.), Investigación en Educación Matemática XI (pp. 121-140). La Laguna: SEIEM.

Gal, I., \& Ginsburg, L. (1994). The role of beliefs and attitudes in learning statistics: towards an assessment framework. Journal of Statistics Education, 2(2). doi: https://doi.org/10.1080/10691898.1994.11910 $\underline{471}$

George, D., \& Mallery, P. (2003). SPSS for Windows step by step: A simple guide and reference. 11.0 update. Boston: Allyn\& Bacon.

Haladyna, T., \& Shaughnessy, J. (1982), Attitudes toward science: A quantitative synthesis. Science Education, 66, 547-563. doi: https://doi.org/10.1002/sce.3730660406

Hilton, S. C., Schau, C., \& Olsen, J. A. (2004). Survey of Attitudes Toward Statistics: Factor structure invariance by gender and by administration time. Structural Equation Modeling, 11(1), 92-109. doi: https://doi.org/10.1207/S15328007SEM1101 7

Hommik, C., \& Luik, P. (2017). Adapting the survey of attitudes toward statistics (SATS-36) for Estonian secondary school students. Statistics Education Research Journal, 16(1), 228-239.

Hood, M., Creed, P. A., \& Neumann, D. L. (2012). Using the expectancy value model of motivation to understand the relationship between student attitudes and achievement in statistics. Statistics Education Research Journal, 11, 72-85.

Hu, L., \& Bentler, P. M. (1999). Cutoff criteria for fit indexes in covariance structure analysis: Conventional criteria versus new alternatives. Structural Equation Modeling: A Multidisciplinary Journal, 6(1), 1-55. doi: https://doi.org/10.1080/10705519909540118

Khavenson, T., Orel, E., \& Tryakshina, M. (2012). Adaptation of survey of attitudes towards estatistics (SATS 36) for Russian sample. Procedia-Social and Behavioral Sciences, 46, 2126-2129. doi: https://doi.org/10.1016/j.sbspro.2012.05.440

Ma, X., \& Kishor, N. (1997). Assessing the Relationship between Attitude toward Mathematics and Achievement in Mathematics: A Meta-Analysis. Journal for Research in Mathematics Education, 28(1), 26-47. doi: https://doi.org/10.2307/749662

Marsh, H. W., Hau, K. T., \& Wen, Z. (2004). In search of golden rules: Comment on hypothesis testing approaches to setting cutoff values for fit indexes and dangers in 
overgeneralizing $\mathrm{Hu} \&$ Bentler's (1999) findings. Structural Equation Modeling, 11, 320-341.

doi:

https://doi.org/10.1207/s15328007sem1103_2

Mills, J. D. (2004). Students' attitudes toward statistics: implications for the future. College Student Journal. 38(3), 349-361.

Nolan, M. M., Beran, T., \& Hecker, K. G. (2012). Surveys assessing students' attitudes toward statistics: A systematic review of validity and reliability. Statistics Education Research Journal, 11(2), 103-123. Retrieved from http://iaseweb.org/documents/SERJ/SERJ11(2) Nolan.pdf

Ntoumanis, N. (2001). A self-determination approach to the understying of motivation in physical education. British Journal of Educational Psychology, 71, 225-242. doi: https://doi.org/10.1348/000709901158497

Onwuegbuzie, A. (2000). Attitudes toward statistics assessment. Assessment \& Evaluation in Higher Education, 25(4). 321339. doi: https://doi.org/10.1080/713611437

Onwuegbuzie, A.J., \& Wilson, V.A. (2003). Statistics Anxiety: nature, etiology, antecedents, effects, and treatments - a comprehensive review of the literature. Teaching in Higher Education, 8, 195-209

Osborne, J., Simon, S., \& Collins, S. (2003). Attitudes towards science: a review of the literature and its implications. International Journal of Science Education, 25(9), 10491079. doi: https://doi.org/10.1080/0950069032000032199

Peters, P., Smith, A., Middledorp, J., Karpin, A., Sin, S., \& Kilgore, A. (2013). Learning essential terms and concepts in statistics and accounting. Higher Education Research \& Development, 33(4), 742-756. doi: https://doi.org/10.1080/07294360.2013.863838

Ramirez, C., Schau, C., \& Emmioglu, E. (2008). The importance of attitudes in statistics education. Statistics Education Research Journal, 11(2), 57-71.

Roberts, D. M., \& Bilderback, E. W. (1980). Reliability and validity of a statistics attitudes survey. Educational and Psychological
Measurement, 40, 235-238. doi: https://doi.org/10.1177/001316448004000138

Ruiz de Miguel, C. (2015). Actitudes hacia la estadística de los alumnos del Grado en Pedagogía, Educación Social y Maestro de Educación Primaria en la UCM. Educación XX1, 18(2), 351-374. doi: https://doi.org/10.5944/educxx1.12158

Schau, C. (2003). Survey of Attitudes Toward Statistics (SATS-36). Retrieved from http://evaluationandstatistics.com/

Schau, C. (2003). Survey of Attitudes Toward Statistics (SATS-36). Retrieved from http://evaluationandstatistics.com/

Schau, C., Stevens, J., Dauphinee, T., \& Del Vecchio, A. (1995). The development and validation of the Survey of Attitudes Toward Statistics. Educational and Psychological Measurement, 55(5), 868-875. doi: https://doi.org/10.1177/0013164495055005022

Sesé, A., Jiménez, R., Montaño, J. J., \& Palmer, A. (2015). ¿Pueden las actitudes hacia la estadística y la ansiedad estadística explicar el rendimiento de los estudiantes? Revista de Psicodidáctica, 20(2), 285-304. doi: https://doi.org/10.1387/RevPsicodidact.13080

Stanisavljevic, D., Trajkovic, G., Marinkovic, J., Bukumiric, Z., Cirkovic, A., \& Millic, N. (2014). Assessing Attitudes towards Statistics among Medical Students: Psychometric Properties of the Serbian Version of the Survey of Attitudes Towards Statistics (SATS). PLoS ONE, 9(11): e112567. doi: https://doi.org/10.1371/journal.pone.0112567

Sulieman, H. (2015). Students' conceptions of statistics: an exploration of attitudes across majors. International Research in Education, 3(2), 158-172. doi: https://doi.org/10.5296/ire.v3i2.7622

Sutarso, T. (1992). Some variables in relation to students'anxiety in learning statistics. Paper presented at the Annual Meeting of the MidSouth Educational Research Association, Knoxville.

Tempelaar, D. T., Schim, S., \& Gijselaers, W. H. (2007). A structural equation model analyzing the relationship of students' attitudes toward statistics, prior reasoning 
abilities and course performance. Statistics Education Research Journal, 6(2), 78-102. Retrieved from http://www.stat.auckland.ac.nz/ iase/serj/SER J6\%282\%29_Tempelaar.pdf

VanHoof, S., Kuppens, S., Castro Sotos, A. E., Verschaffel, L., \& Onghena, P. (2011). Measuring statistics attitudes: Structure of the Survey of Attitudes Toward Statistics (SATS36). Statistics Education Research Journal, 10(1), 35-51. Retrieved from http://iaseweb.org/documents/SERJ/SERJ10(1)_Vanhoo f.pdf

Vilá, R, \& Rubio, M. J. (2016). Actitudes hacia la Estadística en el alumnado del grado de Pedagogía de la Universidad de Barcelona. REDU. Revista de Docencia Universitaria, 14(1), 131-150. doi: https://doi.org/10.4995/redu.2016.5766

Walker, E. R., \& Brakke, K. E. (2017). Undergraduate psychology students' efficacy and attitudes across introductory and advanced statistics courses. Scholarship of Teaching and Learning in Psychology, 3(2), 132-140. doi: https://doi.org/10.1037/stl0000088
Waters, L. K., Martelli, T. A., Zakrajsek, T., \& Popovich, P. M. (1988). Attitudes toward statistics: an evaluation of multiple measures. Educational and Psychological Measurement, 45(2), 401-405.

Wigfield, A., \& Eccles, J. S. (2000). Expectancy-value theory of achievement motivation. Contemporary Educational Psychology, 25(1), 68-81. doi: https://doi.org/10.1006/ceps.1999.1015

Wise, S. L. (1985). The development and validation of a scale measuring attitudes toward statistics. Educational and Psychological Measurement, 45, 401-405. doi: https://doi.org/10.1177/001316448504500226

\section{Agradecimientos}

Los autores agradecen a Candace Schau su autorización para el uso de la escala SATS-36 y la información sobre la utilización de este instrumento aportada en el sitio http://www.evaluationandstatistics.com

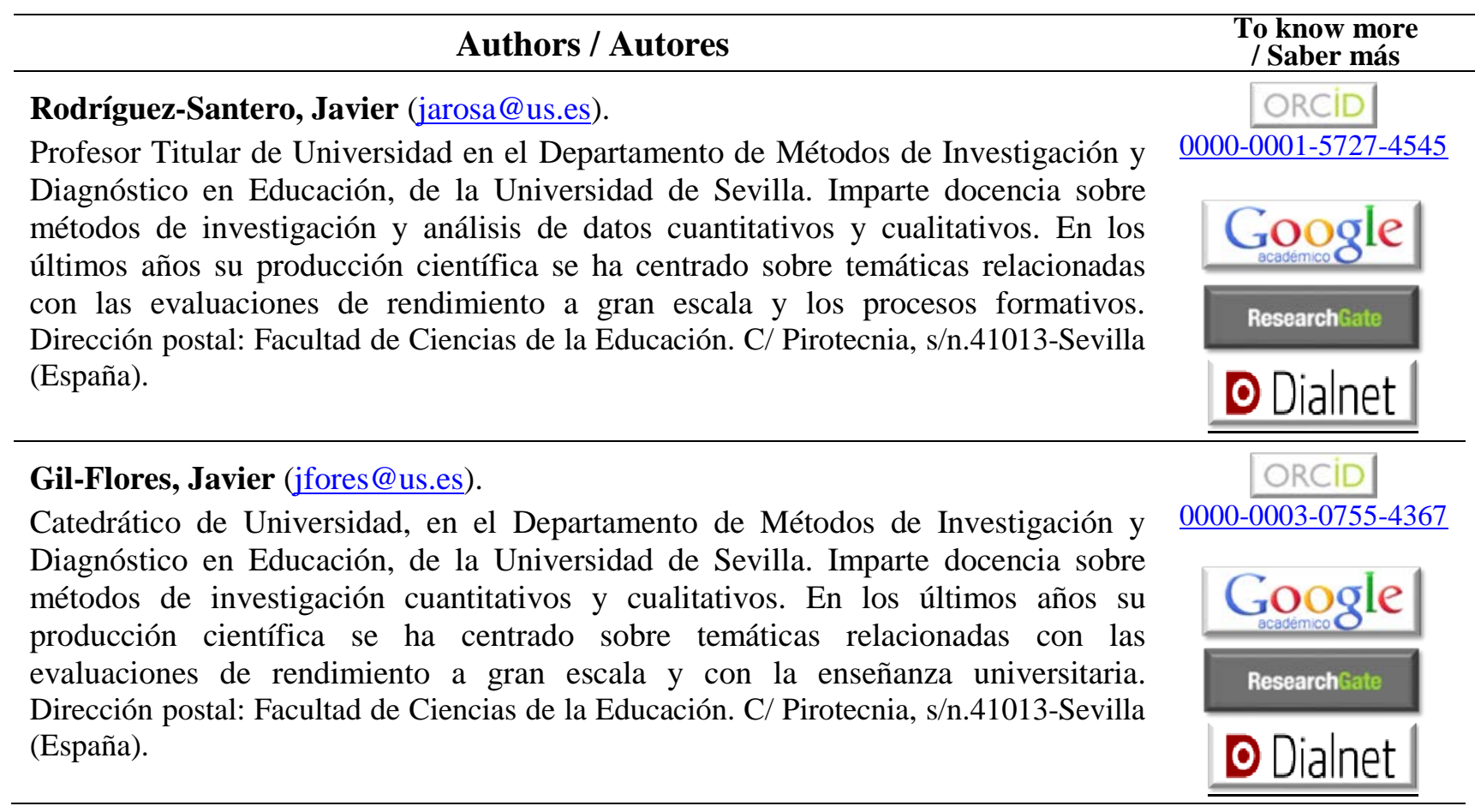




\section{RELIEVE}

Revista ELectrónica de Investigación y EValuación Educativa E-Journal of Educational Research, Assessment and Evaluation

[ISSN: 1134-4032]

\section{(c) (1) (9)}

Esta obra tiene licencia de Creative Commons Reconocimiento-NoComercial 4.0 Internacional.

This work is under a Creative Commons Attribution 4.0 International license. 